\title{
Telephone triage hotlines: Effective screens or open gates?
}

- Cite as: CMAJ 2020 January 13;192:E46-7. doi: 10.1503/cmaj.1095837

Posted on cmajnews.com on December 11, 2019

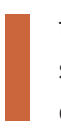

t may seem like teletriage services send everyone to the emergency department, but that's not the case in Saskatoon, according to a recent study. Yet, with limited independent reporting on these services across Canada, it's unclear how much they prevent unnecessary visits, either.

A recent study, published in the Canadian Journal of Emergency Medicine, looked at whether Saskatchewan's 24-hour HealthLine is flooding emergency departments with patients who don't need to be there. According to coauthor Dr. Mark Wahba, many emergency workers believe that teletriage services like HealthLine increase their workload. "You commonly get an eyeroll from the nurse, saying 'HealthLine sent this person in. There's nothing wrong with them. They send everybody here,"' Wahba explains.

Wahba and colleagues cross-referenced HealthLine calls over a 90-day period with patients who registered in any of Saskatoon's three emergency departments within 48 hours of those calls. They found that HealthLine suggested that $17.9 \%$ of 3938 callers go to the emergency department, and just over half followed that advice, accounting for $1.3 \%$ of total patient volume for that period.

"If we didn't have HealthLine, we'd have the potential of all of these callers coming for advice or treatment, so a $17.9 \%$ referral rate seems pretty reasonable," Wahba says.

HealthLine's province-wide referral rate is higher; in 2018/19, the teletriage service directed $28 \%$ of 79393 total callers to go to emergency.

Teletriage services in other provinces report similar rates. Ontario's Telehealth service advised $25 \%$ of all callers in

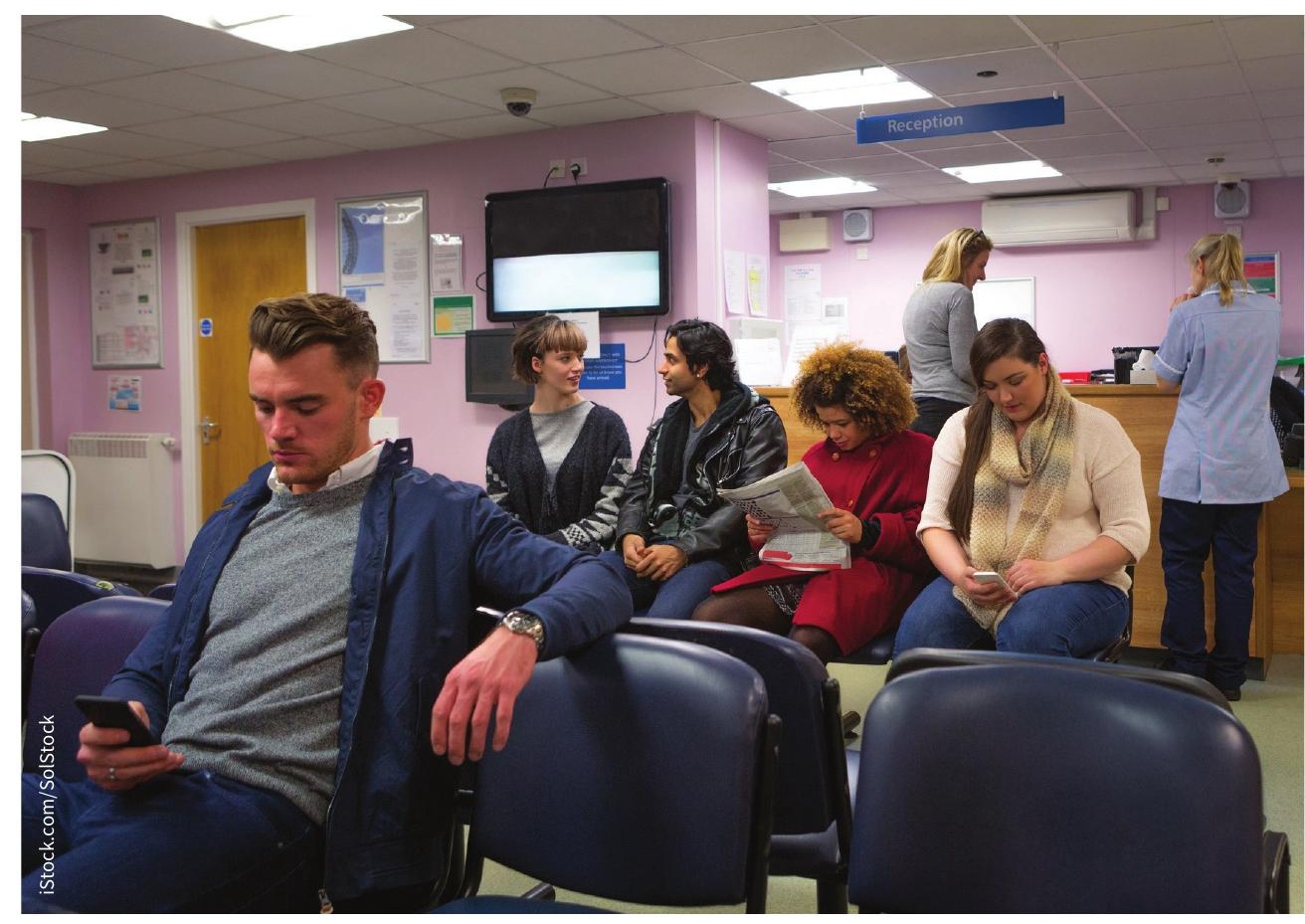

A Saskatchewan study shows the province's teletriage service sent only one in five callers to emergency departments in Saskatoon.

$2018 / 19$ to go to emergency or call 911 , according to a government spokesperson. And in Nova Scotia, an external review of the province's 811 phone line found that it sent $21 \%$ of callers in 2016 to the nearest emergency department.

Wahba and colleagues found that $60 \%$ of people referred by HealthLine to Saskatoon emergency departments underwent diagnostic imaging, laboratory tests or an electrocardiogram, and $66 \%$ received some form of treatment, suggesting that most concerns couldn't be addressed at home or over the phone. The researchers also found that rates of admission to hospital were similar for patients referred by HealthLine and people who visited emergency of their own accord (12\% versus $16 \%$ ).
However, the study didn't explore whether these patients could have received the care they required elsewhere.

That tricky question of appropriateness remains a concern for many emergency workers. Dr. Alan Drummond, co-chair of public affairs for the Canadian Association of Emergency Physicians, says many of the patients he sees referred to emergency by Ontario's Telehealth service could have waited to be seen by a general practitioner. "The usual response is a collective sigh and a mass rolling of the eyes," he says. "That probably says something."

Nova Scotia's external evaluation of its 811 service included a survey of health care professionals, including paramedics, emergency nurses and emergency physicians. 
Fewer than one-third agreed or strongly agreed that 811 service recommendations were appropriate. One in five very rarely or never agreed with the recommendations made by the service. And roughly $80 \%$ of physicians and $60 \%$ of paramedics disagreed or strongly disagreed with recommending the service to patients as an accurate source of information.

According to Heather Fairbairn of Nova Scotia's Department of Health and Wellness, the review found that "people referred to the emergency department were of a higher acuity [than self-referred] and that would indicate the service is working as it should."
But, because it was difficult to quantify how many people were diverted from emergency departments, it remains unclear if the service is worth its $\$ 5.45$ million price tag.

Roberta Wiest, director of Saskatchewan's HealthLine service, acknowledges that the protocols used by teletriage nurses to assess callers do err on the side of caution. "When a registered nurse is assessing somebody over the telephone, she can't see the patient. She can't touch them. She hasn't got any electronic devices to monitor their blood pressure or whatever the case may be. You are assessing someone blind," Wiest says.
According to Toronto emergency physician Dr. Hasan Sheikh, health professionals must also be careful not to let their perceptions of teletriage services affect the quality of the care they provide. "I think that emergency department providers are at a risk of recall bias, where they remember the small number of cases where the patient comes to the emergency department only because they've been told to by telephone triage," he explains. "Therefore, some providers may take the presenting complaints of patients referred by telephone triage less seriously."

Greg Basky, Saskatoon, Sask. 